\title{
Enseignement d'une DNL en langue étrangère : de la clarification à la conceptualisation
}

\section{Laurent Gajo}

\section{(2) OpenEdition}

1 Journals

Édition électronique

URL : http://journals.openedition.org/trema/448

DOI : $10.4000 /$ trema.448

ISSN : 2107-0997

Éditeur

Faculté d'Éducation de l'université de Montpellier

\section{Édition imprimée}

Date de publication : 1 septembre 2007

Pagination : $37-48$

ISSN : 1167-315X

\section{Référence électronique}

Laurent Gajo, « Enseignement d'une DNL en langue étrangère : de la clarification à la

conceptualisation », Tréma [En ligne], 28 | 2007, mis en ligne le 13 octobre 2010, consulté le 01 mai 2019. URL : http://journals.openedition.org/trema/448; DOI : 10.4000/trema.448

Ce document a été généré automatiquement le 1 mai 2019.

Trema 
Enseignement d'une DNL en langue étrangère : de la clarification à la conceptualisation

Laurent Gajo

Introduction 
1 Dans cet article, nous traiterons l'enseignement bilingue comme relevant d'une didactique multi - intégrée, au croisement d'une langue première (L1) et d'une langue seconde (L2), mais aussi de langues et de disciplines non linguistiques (DNL), comme l'histoire, les mathématiques ou la biologie. Ces multiples croisements ne se présentent pas seulement comme un fait, comme une donnée de départ, mais ils s'élaborent dans l'interaction et prennent sens dans le cadre d'un cursus disciplinaire intégrant des enjeux linguistiques.

2 S'ils fondent l'enseignement bilingue, ils sont perçus et exploités de façon variable par les enseignants et par les chercheurs. Nous souhaitons ici en montrer la

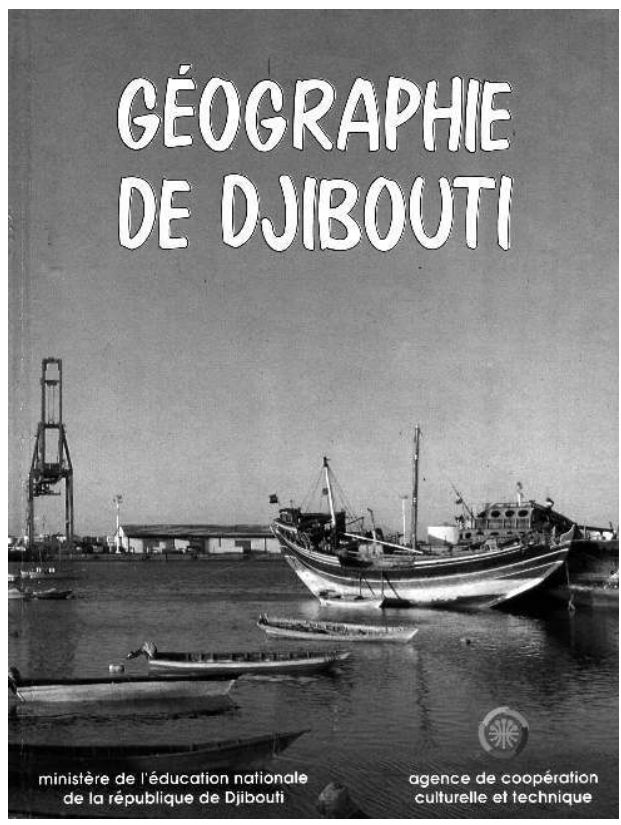
dynamique, avec une réflexion particulière

sur la construction intégrée des savoirs linguistiques et disciplinaires. Nous verrons ainsi comment nous pouvons glisser de la « simple " clarification terminologique à la conceptualisation dans la discipline. Dans ce cadre, nous tenterons de comprendre en quoi l'écran de la L2 et/ou le recours au bi - plurilinguisme peut servir d'atout dans la construction des savoirs.

\section{L'enseignement bilingue, une didactique multi - intégrée}

3 La notion d'intégration occupe une place centrale dans le fonctionnement et la description de l'enseignement bilingue. La plupart des sigles européens utilisés actuellement y font recours : CLIL (Content and Language Integrated Learning, Marsh et al., 2001), EMILE (Enseignement d'une matière par l'intégration d'une langue étrangère, Baetens Beardsmore, 1999), AICL (Apprentissage intégré de la langue et du contenu, Commission Européenne, 2004).

La question de l'intégration présente un intérêt à trois niveaux au moins : cognitif, linguistico - communicatif, sociopolitique - symbolique.

5 Au niveau cognitif, il s'agit de décrire les processus à l'œuvre dans le travail disciplinaire en L2 et / ou en bilingue, et surtout de comprendre les types de savoirs sollicités, d'une part, et construits, de l'autre. Le fait que ces savoirs relèvent tantôt plus du paradigme disciplinaire, tantôt plus du paradigme linguistique revêtira une signification particulière.

6 Au niveau linguistico - communicatif, il est important de saisir les modes d'intégration ou les modalités de contact entre L1 et L2, tant au niveau de la micro - alternance (recours ponctuel et non programmé à l'autre langue) que de la macro - alternance (attribution programmée d'une langue donnée à une portion du curriculum et de la séquence didactique). Duverger (ici même) fournit une distinction supplémentaire entre macro - et 
méso - alternance (ou alternance séquentielle) pour apporter une nuance entre la question du curriculum et celle de la séquentialité didactique.

7 Au niveau sociopolitique ou symbolique, les enjeux de l'intégration apparaissent d'abord en termes d'articulation entre des choix de langues et des choix de disciplines. Si la répartition des langues dans le curriculum relève de ce que nous avons désigné comme « macro - alternance ", il faut bien avoir à l'esprit que celle - ci s'organise à partir de langues plus ou moins chargées contextuellement. De même, les DNL sont reconnues comme plus ou moins porteuses de la réussite scolaire. En amont de la pratique pédagogique gravitent ainsi des représentations variables au sujet des articulations raisonnables, efficaces, souhaitables entre L1, L2 et DNL (Gajo, 2001 et 2005).

Les questions d'intégration peuvent donc se lire à plusieurs niveaux et impliquent, dans le cas de l'enseignement bilingue, le curriculum dans sa transversalité. Il s'agirait alors de décrire les phénomènes de contacts de langues et de disciplines sur deux axes, vertical (contextualisation socio - éducative, représentations environnantes) et horizontal (co textualisation, séquentialisation pédagogique).

\section{Mais où est passée la DNL?}

Dans l'enseignement bilingue, le terrain prioritaire tient aux DNL, qui s'offrent d'une certaine manière au bi - plurilinguisme. Cependant, cette ouverture des DNL ne saurait obéir à une forme d'abandon didactique, où le bénéficiaire serait avant tout extérieur. Si la DNL peut absorber plus ou moins naturellement la diversité des langues et les enjeux particuliers de la L2, c'est qu'elle doit tout aussi naturellement se mouler dans les processus langagiers. Le travail bi - plurilingue trouve ainsi un terrain très favorable en DNL, pour au moins quatre raisons.

Premièrement, la verbalisation se présente comme une condition centrale pour le processus d'enseignement / apprentissage. Même les disciplines qui possèdent un langage formel propre, comme les mathématiques, recourent abondamment à la langue naturelle pour la mise en place et le contrôle des savoirs. La scolarisation se fonde alors sur une sorte de " tout linguistique ", toutefois moins visible et / ou perceptible en L1 qu'en L2. Dans ce sens, il s'agirait de s'interroger sur la pertinence de la désignation " discipline non linguistique ", peu adéquate aux enjeux recherchés par l'enseignement bilingue. L'expression de DNL comporte d'évidents avantages pratiques, mais résiste à une conceptualisation sérieuse. Nous proposerions donc de la troquer contre la notion de discipline dite non linguistique (DdNL).

Deuxièmement, si l'enseignement bilingue prolonge - en la redimensionnant - la « révolution " communicative en didactique des langues, il faut remarquer que la communication à l'école trouve sa cible la plus immédiate et donc authentique dans les savoirs scolaires eux - mêmes (savoirs des DdNL). Nous reviendrons plus bas sur la notion d'authentification.

12 Troisièmement, la $\mathrm{L} 2$ - et le plurilinguisme de manière générale - constitue un outil naturel d'élaboration et d'accès aux savoirs. En effet, la science s'est développée dans différentes cultures et différentes langues, les savoirs ayant en outre subi diverses migrations. L'école monolingue fait écran à cette réalité, en présentant de manière relativement lisse des savoirs structurés souvent dans un grand nombre de ruptures, y compris linguistiques. 

compris en L1) et le travail sur les savoirs disciplinaires. Elle enrichit la boîte à outils pédagogique et peut, à toutes fins utiles, aussi bien complexifier que dénouer des tâches ou des problèmes disciplinaires.

Du côté de l'apprenant, la verbalisation aussi se présente comme un défi majeur. Très vite, il se rend compte qu'il ne peut ni ne doit tout dire dans une seule langue. Le bi plurilinguisme constitue alors aussi bien un moyen qu'un but de la communication à l'école. En même temps qu'il se construit, il offre des ressources disponibles pour la gestion des tâches. L'école constitue un cadre symbolique (image institutionnelle) et pratique (contrat didactique) de légitimation, de construction et de stimulation de ce bi plurilinguisme. L'élève y sera confronté sous différents angles, en termes d'instruction (accès aux savoirs), d'entraînement (lieu de pratique communicative) et d'éducation (mise en circulation de représentations).

\section{De la langue étrangère à la langue « étrange » : vers la défamiliarisation}

Le recours à une L2 dans la scolarisation entraîne à première vue une complexification des situations didactiques, même si l'on considère la pluralité comme une condition ordinaire d'accès aux savoirs. Cette complexification prend plusieurs formes et demande d'abord une description relativement précise. Il s'agit ensuite de ne pas amalgamer complexification et complication et de mesurer au mieux le rapport coûts / bénéfices.

Même si le plurilinguisme correspond à une donnée sociale largement répandue et de plus en plus ressentie comme ordinaire, la situation de travail bi - plurilingue ne va pas toujours de soi pour les acteurs pédagogiques et place l'élève face à des problèmes. Ceux ci favorisent toutefois la décontextualisation des faits langagiers, la réflexivité et induisent une défamiliarisation des formes linguistiques, même en L1. Loin de nuire à l'apprentissage, ces divers processus le stimulent du moment qu'ils sont pris au sérieux dans le cadre didactique. L'opacité de la L2 constitue alors une chance bien plus qu'un obstacle.

Cette chance concerne aussi bien la DdNL que les langues, dans la mesure où l'opacité de la structure discursive du contenu disciplinaire entraîne la déconstruction de l'objet disciplinaire lui-même. Nous parlerons de densité de cet objet (voir BERTHOUD \& GAJO, 2005). On observe ainsi en classe bilingue une continuité de traitement entre l'opacité de la langue et la densité du contenu disciplinaire, et le travail intégré permet l'émergence concomitante des deux types de savoir.

Plus concrètement, on remarque que la reformulation en L1 et en L2 constitue une des bases du travail métalinguistique et peut aboutir à des processus métacognitifs visant le traitement de l'information disciplinaire. Qu'elle agisse au niveau micro ou méso, qu'elle croise l'alternance ou non, qu'elle soit initiée par le maître ou l'élève, la reformulation et ses différentes facettes doivent être (re) pensées dans le cadre de l'enseignement bilingue et de l'enseignement tout court. 


\section{De la reformulation à la conceptualisation}

19 Le processus de reformulation intéresse le contenu disciplinaire, dans la mesure où il contribue à sa clarification et sa transmission. Il demeure cependant plus ancré du côté des savoirs linguistiques et travaille avant tout sur l'opacité du discours.

Dans l'enseignement bilingue, il s'agit de passer du traitement de l'information disciplinaire à la construction, à l'établissement des savoirs en DdNL, de l'opacité du discours à la densité des savoirs. Pour revenir à la question de l'authenticité, au cœur de la compétence de communication, on parlera alors d'authentification (voir HANSE, 2000). L'authenticité consiste à baser la communication et, partant, la pratique pédagogique sur des situations / documents socialement attestés et pertinents ; l'authentification consiste, elle, à montrer que la langue n'existe pas en dehors de contenus, d'enjeux « non linguistiques » qu'elle contribue à façonner et, par conséquent, elle vise à thématiser les faits linguistiques dans le cadre de ces contenus et pour ceux - ci.

L'attention prêtée à l'authentification de la langue, à la densité des savoirs disciplinaires sous-tend le processus de conceptualisation, plus directement dirigé vers la DdNL que ne l'est le processus de reformulation ou, plus généralement, de clarification, ce dernier fonctionnant néanmoins souvent comme déclencheur. Passons à ce stade à l'analyse d'une première séquence, tirée d'une leçon d'histoire dans un lycée bilingue du canton de Vaud (Suisse), où l'allemand fonctionne comme L2.

- Séquence 1

- 17En Wilson. . was finden sie wichtig was finden sie interessant $(1 \mathrm{sec})$ wie $(1 \mathrm{sec})$ ist seine Haltung\

$18 \mathrm{E}$ XXX

$19 \mathrm{E} 15 \mathrm{e}: \mathrm{h}$ die und der Angst vor der Revanche ... und er. . er denkt dass e $: \mathrm{hm} .$. man ehm die eh eh die. das deutsche. Volk . e:h $(1 \mathrm{sec})$ ehm gut e $: \mathrm{h}$

$(2 \mathrm{sec})$ traiter

$(2 \mathrm{sec})$

20En behandeln

21E15 behandelt e:h (?nicht) . . e e:hm um e:hm um sicher zu: . . (? bleiben)

22En ah wenn sie sagen . man muss eh. . eh die Deutschen gut behandeln können sie das ein bisschen genauer erklären \. was heisst gut behandeln \}

23E15 \&eh das heisst eh ge eh. gerecht sein

$(3 \mathrm{sec})$

24En genau \. gerecht sein. die Gerechtigkeit \verstehen . . eh verstehen sie alle dieses Wort/ $(2 \mathrm{sec})$ gerecht wer versteht das nicht das können sie ja sagen dann können wir das klären $\backslash$. o ok

25E \&justice ... juste

26En huh/ ... Gerechtigkeit juste. eh justice huh être juste \Gerechtigkeit (3 sec)

((écrit au rétroprojecteur)) im Friedensvertrag (10 sec) ((écrit au rétroprojecteur)) (? denn) was ist . was passiert wenn eh. e:h ... wenn der Friedensvertrag ungerecht ist $\backslash$ was wäre ungerecht zum Beispiel\

$27 E 15$ e:h. eh zu viel Geld e:h 28En ja genau $\backslash$ zu viel Geld zu bezahlen zu hohe Reparationen. zu e:h bezahlen das wäre vielleicht ungerecht was könnte. was noch ungerecht sein $\backslash[. .$.

(2.1002.G5.12.Hi.D.1) 

comportement (Haltung) de Wilson, l'élève 15 bute en 19 sur le terme traiter, qu'il n'arrive pas à exprimer en allemand. Il produit un énoncé hésitant et, après une pause de 2 secondes, passe à la L1. L'enseignant lui fournit ensuite, en 20, l'équivalent en allemand ( behandeln), en n'hésitant pas à recourir à la traduction, sorte de reformulation. L'élève reprend aussitôt la forme proposée (behandelt), en l'intégrant directement dans son contexte discursif (recontextualisation). Il démontre ainsi sa maitrise linguistique et la disponibilité du terme behandeln pour la communication.

Lenseignant ne souhaite toutefois pas en rester là et prolonge la réflexion autour de behandeln à partir de 22. Il fait référence au discours de l'élève (wenn sie sagen - quand vous dites) et lui demande d'expliquer plus précisément (genauer erklären), en décontextualisant progressivement l'expression die Deutschen gut BEHANDELN (avec accent d'insistance sur le verbe) - 1er mouvement d'abstraction), qui devient was heisst gut behandeln (que signifie bien traiter) - 2e mouvement d'abstraction. Il ne s'agit dès lors plus tellement d'assurer les moyens de la communication, mais d'authentifier la langue dans le savoir historique et d'en étayer ainsi la densité. En 23, l'élève 15 propose l'expression gerecht sein (être juste), validée par l'enseignant en 24 avant d'être nominalisée (Gerechtigkeit). Notons que le processus de nominalisation, bien que de nature linguistique, joue un rôle souvent important en rapport avec la conceptualisation, les concepts (savoirs) correspondant en principe à des noms.

Mais là encore, le terme Gerechtigkeit (ou gerecht) n'est peut-être pas disponible pour la communication (verstehen sie alle dieses Wort - comprenez-vous tous ce mot) et l'on repart en direction de l'opacité avec une volonté de clarification (klären). Dans ce mouvement, la L1 est de nouveau sollicitée, d'abord par un élève et ensuite par l'enseignant. Une fois ce savoir linguistique établi, on revient plus directement à la DdNL avec, pour fixer les notions / concepts, une demande d'exemples (was wäre ungerecht zum Beispiel - qu'est-ce qui serait injuste par exemple). Cette demande fait suite à une première formulation, où le terme central est accentué (UNgerecht) sur la syllabe potentiellement problématique (unindique le contraire). Il s'agit toutefois d'une aide « en passant » de l'enseignant, qui ne thématise pas une question linguistique que les élèves doivent pouvoir gérer sans difficulté sur la base de leurs savoirs linguistiques déjà disponibles.

Cette séquence illustre bien les mouvements de bascule entre une attention portée sur l'opacité et une attention portée sur la densité. Parfois, les deux mouvements ne sont pas seulement en immédiate continuité mais se trouvent superposés ou intercalés. On en remarque toutefois bien la nature sensiblement différente, et l'allemand, à travers les deux termes klären et erklären, nous aide à voir à la fois la continuité et la différence entre les processus de clarification et d'explication (conceptualisation).

La clarification précède souvent, en la solidifiant, la conceptualisation, d'autant plus en L2. Par son opacité évidente, celle - ci offre alors des ressources utiles au processus de conceptualisation. Pour rendre mieux le traitement continu des deux processus et le rôle particulier de la L2, nous proposons de mettre en parallèle les notions de re - médiation et de médiation. Cette dernière relève de l'existence même de la langue ou de tout système de médiation symbolique (voir GROSSEN \& PY, 1996), par lequel s'organisent les 
savoirs. Le lexique y joue un rôle particulier (voir LÜDI, 1994), mais les autres unités de la communication ne sauraient être négligées. Le processus de catégorisation de même que celui de désignation peuvent être ramenés à la médiation. Nous pouvons aussi y ranger la nominalisation, que nous venons d'évoquer à travers l'analyse de la séquence. Concernant la re - médiation, elle doit être entendue aussi bien comme une deuxième médiation (par l'usage de la L2) que comme un ensemble de stratégies de résolution de problèmes (sens de remédier) ou d'ajustement des ressources communicatives. En relèveraient la reformulation et tout ce qu'il est convenu d'appeler les activités métalinguistiques.

Nous aboutissons ainsi au schéma suivant.

\begin{tabular}{|l|l|l|}
\hline $\begin{array}{l}\text { Savoir linguistiques } \\
\text { (paradigmes) }\end{array}$ & $\begin{array}{c}\text { Discours } \\
\text { (tâche) }\end{array}$
\end{tabular}

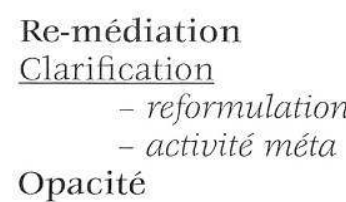

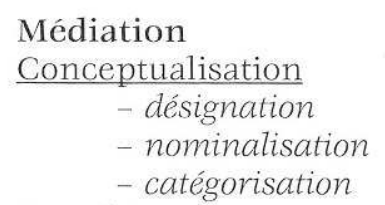

Opacité

Dans l'enseignement bilingue, le traitement des questions de médiation peut le plus souvent se réaliser suite à des problèmes de re - médiation. Ceux-ci contribuent à rendre celles - là visibles et pertinentes pour la construction des savoirs, ce qui n'est pas le cas en classe monolingue, où la médiation n'apparaît pas en tant que telle et ne donne donc pas lieu à un travail explicite de déconstruction. Pour illustrer davantage ce travail continu entre médiation et re - médiation, nous prenons maintenant l'examen d'une deuxième séquence.

- Séquence 2

- 66En [...] alors ensuite \. vous allez parler. des MOUVEMENTS. c'est parfait. " mouvements » $X$ donner tout de suite parce que vous l'avez déjà dit. le mouvement $(4,5 \mathrm{sec})$ et peut-être que vous avez (encore) un autre verbe que vous pouvez reconnaitre. si vous pensez. à ... X en polonais. la machine qui: si vous prenez le train. la machine qui tire le train en polonais/ $(3 \mathrm{sec}$ ) ciuchcia (la locomotive - argot enfantin)

67ElèvesX

68Enalbo/. pociag (ou bien. le train)

69Elèvepociag (le train)

70Enpociag (le train) en français la /

71Elèvelokomotywa (la locomotive)

72Enlokomotywa (locomotive) en français locomotion la locomotive/ très bien. la locomotive. le mouvement. c'est autrement c'est la locomotion $\backslash(3 \mathrm{sec}) \mathrm{XX}$ ce qu'on a dit avant po prostu bysmy przetlumaczyli jako (nous allons le traduire tout simplement comme ...? 73Elève (ruch)/ (le mouvement)

74Enruch oczywiscie ruch \(le mouvement, bien sûr, le mouvement) ... donc ici vous avez deuX verbes: vous avez deux termes que vous pouvez utiliser X noms XXX le mouvement la locomotion . et le verbe /. on a déjà XX compliqué. le verbe $\mathrm{X}$ tout ça c'est / 75Elève (? se déplacer) 
76Ense déplacer. oui. une plante on peut pas dire qu'elle se déplace. c'est trop dire déjà. la plante elle ne se déplace pas tellement mais elle/ $(4 \mathrm{sec})$ ça commence par «b» $(3 \mathrm{sec}) \mathrm{XXX}$ bou- : /

77ElèveX

78En « bouger ». «bouger ». donc c'est bon. «bouger ». en plus XXX ce qu'on dit facilement ... parce que la plante. on peut dire qu'elle bouge. elle se tourne vers la lumière. elle bouge vers la lumière. mais elle ne se déplace pas $\backslash$. pour nous c'est bon. le « déplacement » c'est bon $\backslash$. alors deux. quatre. quatre pour le moment. cinquième fonction vitale /

Nous nous trouvons ici dans un collège bilingue de Varsovie, en 2e classe (les élèves ont entre 13 et 14 ans) dans un cours de biologie. Si le recours à L1 revêt plusieurs fonctions, notons tout de même que le système polonais impose aux enseignants de dispenser les notions de DdNL systématiquement en L1 aussi, dans la mesure où les élèves des sections bilingues peuvent être amenés à passer un examen de DdNL en L1. Dans cette séquence, on aborde le chapitre des fonctions vitales, et en particulier la fonction du mouvement. Le terme correspondant semble être disponible pour la communication et l'enseignante le met en évidence en 66. Elle enjoint pourtant les élèves à trouver un autre terme. Le statut de ce terme en lien avec la fonction de médiation ou de re - médiation n'est pas clair à ce stade, mais on sait que les élèves sont capables de le reconnaître en pensant au polonais et qu'il présente donc un rendement sans doute intéressant à ce niveau - là.

On le voit en 72, le terme recherché est locomotion mais, pour y arriver, il faut passer par le polonais lokomotywa, très certainement disponible dans le lexique des élèves et en relation de grande transparence avec le français locomotive. Cette disponibilité n'est toutefois pas immédiate et passe, sous l'impulsion de l'enseignante, par les termes ciuchcia et pociag. Jusque - là, le travail de négociation linguistique relève avant tout de la re - médiation, car il s'agit d'établir les moyens nécessaires ou tout au moins adéquats pour la communication. Notons que ce travail peut opérer au sein même de la L1, en proposant par exemple une réflexion sur les registres (ciuchcia et lokomotywa dans notre séquence).

Le terme de locomotion offre ainsi une alternative à celui de mouvement (le mouvement. c'est autrement la locomotion en 72, vous avez deux termes que pouvez utiliser en 74), même si ce dernier semble moins élaboré, plus disponible parce que, sans doute, moins spécialisé ( nous allons le traduire tout simplement comme en 72). On aurait pu amorcer à ce stade un travail sur la médiation, mais il faut attendre le passage aux verbes (et le verbe en 74) pour s'en approcher. Cette manipulation morpholexicale est présentée comme compliquée par l'enseignante, les verbes directement apparentés aux noms locomotion et mouvement étant soit inexistants soit peu disponibles. Un élève propose cependant se déplacer en 75. En 76, l'enseignante valide ce terme en limitant néanmoins sa pertinence, dans la mesure où il ne s'applique pas aux plantes. Ce terme se montre conceptuellement trop riche, trop spécialisé (c'est trop dire déjà), alors que la fonction vitale à désigner concerne tous les êtres vivants, végétaux compris. Les élèves sont alors invités à trouver un autre verbe, qui commence par b. Il s'agit du verbe bouger, prononcé plusieurs fois par l'enseignante en 78. Cette dernière explicite avec insistance que la plante bouge, se tourne vers la lumière, bouge vers la lumière, mais ne se déplace pas. Le fait de se déplacer est réservé à l'être humain (pour nous c'est bon) et, plus généralement, aux animaux. La différence entre bouger et se déplacer s'explique ici dans le cadre du paradigme disciplinaire, qu'elle contribue à établir. La négociation se place clairement du côté de la médiation, comme 
elle aurait pu (dû) l'être juste avant, pour le travail sur les substantifs. Au sein de la (micro-) séquence de médiation peut toutefois s'insérer un travail de re-médiation, basé par exemple sur la reformulation. C'est le cas ici en 78 quand l'enseignante passe de elle se tourne vers la lumière à elle bouge vers la lumière. L'enchâssement des différentes séquences de travail devrait donner lieu à des descriptions plus précises, qui dépassent toutefois les ambitions du présent article. De même, l'on peut distinguer, aussi bien du côté de la médiation que de la re - médiation, différents types de savoirs linguistiques en fonction de leur caractère plus ou moins lié aux besoins immédiats de la tâche. Nous renvoyons pour cela à GAJO $(2006,2007)$.

L'enseignement bi - plurilingue, pour produire tous ses effets, demande une grande vigilance linguistique de la part des acteurs pédagogiques. La disponibilité de plusieurs langues permet alors à l'enseignant d'engager des stratégies profitables pour sa discipline, nous l'avons vu, mais aussi pour chacune des langues, y compris la L1. Nous en donnons une dernière illustration.

- Séquence 3

- 1En Angela c'est clair/

2E oui

3En ouais ( $1 \mathrm{sec}$ ) weil es ein /. comment est-ce qu'on dit en français/ ( $3 \mathrm{sec}$ ) vous savez/ $(2,5 \mathrm{sec})$ et pis en allemand

$4 \mathrm{E} 1$ gleich $-\mathrm{X}$

5 En gleichschenklig ja $\backslash .$. en français c'est ISOCÈLE (1,5 sec) I-SO-CÈLE \. [...]

6E2 XX en allemand XX quoi ((rires des camarades))

7En vous savez que l'allemand .. est une langue. plus technique. hein célio qui est confortablement installé ((rires)) XX

8 En (céline) vous savez ce que c'est qu'une médiatrice \

$9 \mathrm{E} 3$ ouais. mais

10En et pis une médiane/

$11 \mathrm{E} 3$ ouais $(2,5 \mathrm{sec})$

12En c'est quoi une médiane/

13E3 ((accent alémanique)) c'es : : $t$. une droite qui sépare XX morceaux X ( $5 \mathrm{sec})$

$14 \mathrm{En}$ et pis la médiatrice/

$15 \mathrm{E} 3 \mathrm{X}$ un angle $(5 \mathrm{sec})$

$16 \mathrm{En}$ euh ouais $=$ et ben $=$ écoutez cette confusion est. très très fréquente aussi. . c'est

« bissectrice » qui PARtage un angle en deux. BI. SECTEUR $\backslash$. non mais. je aussi fais cette parenthèse. une digression ... l'allemand est une langue plus technique. parce que ... le mot qui décrit « bissectrice » « médiane » et caetera .. décrit CLAIREMENT ce que c'est \ «bissectrice ». c'est « WINKELHALBIEREND » \... « winkel ». « angle ». « halbierend » « partager en deux » ben . l'allemand =. l'alémanique sait tout de suite et puis « médiatrice ».. l'allemand sait tout de suite. «mittel-. Senkrecht». « milieu ». « perpendiculaire » voilà. c'est pourquoi les maths. c'est plus facile en allemand $\backslash$

(2.1901.B1.S2.Phy.FBi.1)

Nous sommes ici dans une classe de physique au niveau du lycée à Biel - Bienne, ville bilingue de Suisse. On y pratique régulièrement ce que l'on appelle un bilinguisme 
alterné, c'est 6 à - dire une situation scolaire où des élèves des deux communautés linguistiques majoritaires sont représentés et où la langue d'enseignement est alternativement L1 pour les uns et L2 pour les autres. L'enseignant, par exemple en 3, sollicite l'alternance des langues, sans doute pour garantir une terminologie équilibrée dans les répertoires, mais surtout pour renforcer la conceptualisation dans sa discipline. Ce travail sur la langue s'effectue alors dans trois directions :

- à l'intérieur du répertoire germanophone : la décomposition morpholexicale autorise un accès très direct aux concepts ;

- à l'intérieur du répertoire francophone : le même type de manipulation permet à l'enseignant d'éveiller la conscience métalinguistique des francophones, mais avec une productivité moindre pour la DdNL (l'exercice se limite à bissectrice - BI. . SECTEUR, avec de surcroît un degré relatif de transparence conceptuelle);

- au croisement des deux répertoires : les deux langues sont explicitement mises en comparaison, avec l'argument que l'allemand est plus technique que le français.

Ce travail intra - et inter - linguistique profite au développement des divers savoirs, et se dirige en particulier vers la discipline. On remarquera toutefois que l'enseignant base son argumentation sur la transparence linguistique (décrit CLAIREMENT, en parlant de l'allemand en 16). L'allemand serait plus technique car il réduirait le chemin vers le concept, la transparence linguistique permettant d'accéder à la densité conceptuelle. Si l'argument se laisse discuter, il sert la bonne cause et produit sans doute des apprentissages pertinents. Dans cette dynamique, le rapprochement des langues en présence, leur mise en contraste, les traductions ponctuelles ne nuisent en rien à la progression disciplinaire et à l'orientation communicative. Bien au contraire, tout ceci amène des reformulations, des doubles formulations qui, à leur tour, amorcent un processus de conceptualisation.

\section{Conclusion}

L'enseignement bilingue offre une alternative didactique forte, non seulement pour les langues, mais aussi pour les DdNL. Plus encore, il se présente comme une alternative éducative de fond, qui réinterroge le territoire scolaire, les responsabilités linguistiques des acteurs pédagogiques et la réalité sociale. Il ne reconnaît pas seulement la diversité, mais encourage des stratégies de diversification, en prenant au sérieux le contact de langues, didactisé dans sa rencontre avec les disciplines. Il met au centre l'idée d'intégration et regarde les exigences du discours de manière à la fois spécifique et transversale. Il fait face à la complexité, en génère parfois, mais veille à ne pas compliquer.

Son évaluation tout comme son amélioration demande des descriptions très précises, qui permettent d'en établir les contours. Pour cela, on s'appuiera notamment sur des théories de la compétence plurilingue (voir par exemple COSTE, MOORE \& ZARATE, 1997) et / ou du contact de langues (voir par exemple Castellotti, 2001), ou encore sur les études de cas (voir par exemple Cavalli, 2005). Parler d'intégration entre paradigmes de savoirs exige ainsi de ne pas se contenter du niveau macro, mais de descendre aux niveaux méso et micro. Les mécanismes fins du travail intégré s'observent au moyen de supports et d'outils adéquats. Leur variabilité et leur complexité doivent être explicitées et théorisées, d'une part pour montrer aux enseignants expérimentés ce qu'ils pratiquent 
déjà avec succès (explicitation, légitimation), d'autre part pour former les enseignants débutants à une méthodologie particulière (sensibilisation, initiation). Celle - ci prend place au sein d'une didactique du plurilinguisme, relève de ce que nous avons désigné comme une didactique multi - intégrée et ne saurait être appréhendée par des extrapolations trop rapides en provenance de la seule didactique des langues.

$$
\text { Nis }
$$

$\bigwedge$ Intonèmes ascendant et descendant

... ... Pauses de moins d'une seconde (environ _,_et_ de sec.)

(3 sec.) Indication des pauses au - delà de la seconde

: Allongement syllabique

[ Chevauchement

\& Enchaînement rapide

pe=petit Troncation

$<$ ryb $>$ Transcription phonétique

((éternue)) Commentaire du transcripteur

(en)fin Articulation relâchée, segment non prononcé

MAL au CCEUR Accentuation; focalisation, emphatisation prosodiques

(allait - avait) Transcription incertaine

X XX XXX Segments incompréhensibles

\section{BIBLIOGRAPHIE}

BAETENS BEARDSMORE, H. (1999). Consolidating experience in plurilingual education. In MARSH, D. \& MARSLAND, B. (Eds.), CLIL Initiatives for the Millennium (pp. 24-30). Univ. of Jyväskylä: Continuing Education Centre.

BERTHOUD, A.-C. \& GAJO, L. (2005). Traitement des arguments et de leur mise en texte dans le processus de rédaction conversationnelle: continuité entre objets linguistiques et non linguistiques. In BOUCHARD, R. \& MONDADA, L. (éds), Le processus rédactionnel, L'Harmattan.

CALVÉ, P. (1988). Immersion: How High Will the Balloon Fly? Réflexions sur une aventure pédagogique. In: CALVÉ, P. (éd.), Aspects of/de l'immersion, Toronto: Conseil ontarien de recherches pédagogiques.

CASTELLOTTI, V. (2001). La langue maternelle en classe de langue étrangère. Paris : CLE International.

CAVALLI, M. (2005). Education bilingue et plurilinguisme. Le cas du Val d'Aoste. Paris : Didier, coll.

LAL.

Commission européenne (2004), Promouvoir l'apprentissage des langues et la diversité linguistique, Plan d'action « langue » 2004-06.

COSTE, D., MOORE, D. \& ZARATE, G. (1997). Compétence plurilingue et pluriculturelle. Strasbourg: Conseil de l'Europe.

DUVERGER, J. (2007). Didactiser l'alternance des langues en cours de DNL. Ici même.

GAJO, L. (2001). Immersion, bilinguisme et interaction en classe. Paris: Didier, coll. LAL. 
GAJO, L. (2005). Le français langue seconde d'enseignement : choix de modèles, de langues et de disciplines. In LALLEMENT, F., MARTINEZ, P. \& SPAËTH, V. (éds). Français langue d'enseignement, vers une didactique comparative. Le français dans le monde, no spécial, janvier 2005.

GAJO, L. (2006). Types de savoirs dans l'enseignement bilingue: problématicité, opacité, densité. Education et sociétés plurilingues20, 75-87.

GAJO, L. (2007). Linguistic Knowledge and Subject Knowledge: How Does Bilingualism Contribute to Subject Development? The International Journal of Bilingual Education and Bilingualism, Vol. 10, No. 5.

GROSSEN, M. \& B. PY (éd.) (1996). Pratiques sociales et médiations symboliques. Berne : Peter LANG.

HANSE, P. (2000). Les nécessaires articulations entre L1, L2 et disciplines non linguistiques en L2. Le français dans le monde. Recherches et applications, no spécial coordonné par J. DUVERGER, Actualité de l'enseignement bilingue.

LÜDI, G. (1994). Dénomination médiate et bricolage lexical en situation exolingue. Aile 3, 115-143.

MARSH, D. et AL. (2001). Profiling European CLIL Classrooms. Languages open Doors. Finland and Netherlands: University of Jyväskylä and European Platform for Dutch Education.

\section{RÉSUMÉS}

Le présent article aborde la question de l'enseignement bilingue sous l'angle de la construction intégrée des savoirs linguistiques et disciplinaires. Il vise à décrire finement le processus d'intégration par l'analyse discursive de pratiques de classe dans le cadre de différentes DNL. Il montre comment le travail de reformulation (clarification) peut déboucher sur un travail de conceptualisation, comment l'opacité de la L2 peut rencontrer la densité des savoirs disciplinaires. L'idée même de DNL y est critiquée.

European experiments in bilingual education are commonly called content and language integrated learning, although the concept of integration is little described in research and insufficiently perceived in practice. This article aims at a better understanding of the integration process at the crossroads between the linguistic and the subject knowledge. The discourse analysis of several classroom sequences shows the links between clarification and conceptualisation, between the opacity of the L2 and the density of the subject matter.

\section{INDEX}

Mots-clés : clarification, conceptualisation, densité, DNL, enseignement bilingue, intégration, opacité, reformulation, savoir

Keywords : content and language integrated learning (CLIL), density, integration process, opacity, rephrasing

\section{AUTEUR}

\section{LAURENT GAJO}

Université de Genève, Ecole de langue et de civilisation françaises 